\title{
A Clinical Study of Etiopathogenesis And Management of Epistaxis
}

\author{
Dr. Binod Kumar Sinha ${ }^{1}$,Dr. (Mrs.) Chandrakanti Birua ${ }^{2}$,Dr. Dheeraj Kumar ${ }^{3}$ \\ Assistant Professor, Department Of Otorhinolaryngology, Rajendra Institute Of Medical Sciences, Ranchi. \\ Associate Professor, Department Of Otorhinolaryngology, Rajendra Institute Of Medical Sciences, Ranchi. \\ Junior Resident, Department Of Otorhinolaryngology, Rajendra Institute Of Medical Sciences, Ranchi.
}

\begin{abstract}
:
Introduction: Epistaxis is one of the commonest medical emergencies. It affects all age groups and both sexes. The areas for epistaxis are the Little's area and the Woodruff's plexus. The causes are divided into local or general causes. Trauma is considered to be a major etiological factor. Various treatment protocols are utilized to control epistaxis depending upon the type severity and cause of bleeding. The present work was undertaken to study the etiopathogenesis and management of epistaxis by non-surgical and surgical approaches.

Material and Method: Fifty patients of both sexes and all age groups presenting to the Emergency Department or the ENT Outpatient Department at Rajendra Institute of Medical Sciences, Ranchi were taken up for the study. Priority was given to arrest the bleeding and to improve the general condition of the patient. Once the bleeding was controlled, detailed clinical examination and investigations were done to arrive at the diagnosis. Then the patient was given definite treatment depending upon the cause.

Results: Males were affected twice more than females (1.9:1). The age distribution was bimodal with peaks below 20 years and above 40 years. The most common cause was found to be trauma followed by hypertension. Anterior nasal bleeding was noted in majority of the patients. $86 \%$ of the patients were managed by nonsurgical measures; only few (14\%) requiring surgical intervention. Anterior nasal packing was the most effective method of controlling anterior epistaxis; while posterior bleeding was controlled by posterior nasal packing or with Foley's catheter.

Conclusion: Epistaxis has a bimodal age distribution and affects males more than females. Anterior bleeding is more common than posterior bleeding. Trauma is an important etiological factor followed by hypertension. Majority of cases are manageable by conservative measures and only few require surgical intervention.
\end{abstract}

Keywords: Epistaxis; Emergency; Trauma; Nasal packing; Hypertension

\section{Introduction}

Epistaxis means bleeding from the nose. It is derived from the word 'Epistazo' where epi means 'above' and stazo means 'to fall in drops'. 'It is a common clinical condition-encountered by the otorhinolaryngologist. Key clinical areas of epistaxis are the Little's area and the 'Woodruff's plexus. Little's area lies in the anteroinferior part of septum; a common site of anterior epistaxis in children and young adults. ${ }^{2}$ Woodruff s plexus lies just inferior to the posterior end of inferior turbinate; gives rise to posterior, epistaxis in adults. ${ }^{3}$

The causes of epistaxis are numerous which can be divided into local and general causes. Common local causes are Trauma, Infections, Foreign bodies, Deviated nasal septum, Neoplasms. General causes are Hypertension, Blood dyscrasias, Chronic liver disorders, Chronic kidney diseases, Overuse of salicylates and anticoagulants. ${ }^{2}$

The treatment can be separated into two groups-non-surgical and surgical approaches. The non-surgical approaches include local cauterization, anterior and posterior packing. Surgical approaches include arterial ligation, nasal septal surgery and arterial embolization. ${ }^{3}$

Hippocrates $(500 \mathrm{BC})$ was the first to appreciate that pressure on the alae nasi was an effective method of controlling nose bleeds. He was the first to describe vicarious menstruation. ${ }^{2}$ Ali Ibn Rabban Al-Tabiri (850 AD) devoted a chapter of his massive work "The Paradise of Wisdom" to epistaxis. He wrote that "the complaint of nose bleeding is due to swelling of a vein and its rupture, or perhaps on reduction in the force which confines the blood within'.2

In 1769 Morgagni recognized 'the extremely turgid blood vessels about a finger's breadth more or less from the bottom of the nostril'. He was reported to have stopped nose bleeds by introducing his finger and 'pressing that part whereupon the blood ceased to flow.' He also noted that nasal hemorrhage might be arterial in origin for it was his practice 'to syringe the nose with cold water and to apply the spirit of wine to contract the mouths of swollen arteries'. 


\section{Materials And Methodology}

Fifty patients with epistaxis presenting to the Emergency Department on the ENT Outpatient Department at Rajendra Institute of Medical Sciences, Ranchi were taken up for the study. The method used was simple random sampling.

As soon as the patient presented to the hospital, priority was given to arrest the bleeding and to improve the general condition of the patient. Suction of the nasal cavity was done to localise the site of bleeding. Where the bleeding was from Little's area, the site was cauterized with $15 \%$ silver nitrate or $50 \%$ trichloro acetic acid (TCA); where the site was not localised and the patient presented with anterior epistaxis, anterior nasal packing was done with Betadine and light liquid paraffin mixed ribbon gauze or merocel packs. In cases of continued bleeding or posterior epistaxis or both, post nasal packing with Foley's catheter was done. Once the bleeding was controlled, detailed clinical history and examination was carried out.

\section{Inclusion criteria:}

Fifty patients of both sexes and all age groups presenting with epistaxis are included in this study.

Exclusion criteria

Patients presenting with epistaxis resulting from recent septal or paranasal sinus surgery are excluded from this study.

\section{Results}

Statistical Analysis: The data was analyzed by using SPSS 20 software. The data is presented in percentages, rates and ratios. Chi square test was used to find the association between attributes.

Age distribution: The age of the patients varied from 2 to 70 years. The age incidence was more in the 1st and 2 nd decades and then again increased from 4 th decade onwards. The maximum number of cases was in the age group 40-50 years $(28 \%)$.

Table - I: Age distribution

\begin{tabular}{|c|c|c|}
\hline Age group (years) & Number of cases & Percentage \\
\hline $0-10$ & 04 & 8 \\
\hline $11-20$ & 11 & 22 \\
\hline $21-30$ & 03 & 6 \\
\hline $31-40$ & 06 & 42 \\
\hline $41-50$ & 20 & 6 \\
\hline $51-60$ & 03 & 4 \\
\hline$>60$ & 02 & 100 \\
\hline Total & 50 & . \\
\hline
\end{tabular}

Sex distribution: In our study, out of 50 patients, 17 were females and 33 were males with the ratio of 1.94:1 in favour of male.

Table - II: Sex distribution

\begin{tabular}{|c|c|c|}
\hline Sex & Number of cases & Percentage \\
\hline Male & 33 & 66 \\
\hline Female & 17 & 33 \\
\hline
\end{tabular}

Seasonal distribution: in our study maximum number of cases presented during winter season

Table - III: Seasonal distribution

\begin{tabular}{|c|c|c|}
\hline Season & Number of cases & Percentage \\
\hline Winter & 29 & 58 \\
\hline Summer & 12 & 24 \\
\hline Monsoon & 09 & 18 \\
\hline
\end{tabular}

Etiology: In this study the etiology of epistaxis was divided into local and general causes. The local causes included trauma, neoplasms, infection, septal abnormalities and idiopathic. The general causes were hypertension and bleeding disorders. Traumatic causes were accident (22\%), assault (10\%), foreign body (4\%) and nose picking $(6 \%)$. Infectious causes included rhinosinusitis $(2 \%)$, atrophic rhinitis (2\%) and nasal myasis $(4 \%)$. Neoplastic causes comprised of bleeding septal polyps (2\%) and nasopharyngeal angiofibroma (2\%). Other causes were deviated nasal septum $(6 \%)$ and idiopathic $(4 \%)$. The general causes were hypertension $(30 \%)$ and bleeding disorders consisting of hemophilia (2\%) and ITP (2\%).

Table - IV: Etiology

\begin{tabular}{|c|c|c|}
\hline Etiology & Number of cases & Percentage \\
\hline Trauma & 22 & 44 \\
\hline Idiopathic & 02 & 4 \\
\hline Infection & 04 & 8 \\
\hline Neoplasms & 02 & 4 \\
\hline
\end{tabular}


A clinical study of etiopathogenesis and management of epistaxis

\begin{tabular}{|c|c|c|}
\hline DNS & 03 & 6 \\
\hline Hypertension & 15 & 30 \\
\hline Bleeding disorder & 02 & 4 \\
\hline
\end{tabular}

Site of bleeding: In our study, $72 \%$ of patients had anterior nasal bleeding, $16 \%$ had posterior bleeding and $12 \%$ had both.

Table - V: Site of Bleeding

\begin{tabular}{|c|c|c|}
\hline Site & Number of cases & Percentage \\
\hline Anterior & 36 & 72 \\
\hline Posterior & 08 & 16 \\
\hline Both & 06 & 12 \\
\hline
\end{tabular}

Treatment: Out of the non-surgical methods used, medical measures (48.8\%) and anterior nasal packing (34.8\%) contributed the maximum. $86 \%$ of the cases were managed by non-surgical measures as opposed to only $14 \%$ who required surgical intervention.

Table - VI: Treatment (Non Surgical) N = 43

\begin{tabular}{|c|c|c|}
\hline Treatment & Number of cases & Percentage \\
\hline Medical & 21 & 48.7 \\
\hline Cautery & 03 & 7 \\
\hline ANP & 15 & 34.3 \\
\hline PNP & 02 & 4.7 \\
\hline FB removal & 02 & 4.7 \\
\hline
\end{tabular}

Table - VII: Treatment (Surgical) N =7

\begin{tabular}{|c|c|c|}
\hline Treatment & Number of cases & Percentage \\
\hline Septoplasty & 03 & 42.9 \\
\hline Reduction of nasal bone fracture & 02 & 28.6 \\
\hline Excision with electrocautery & 01 & 14 \\
\hline Lateral rhinotomy with ECA ligation & 01 & 14 \\
\hline
\end{tabular}

\section{Discussion}

Age distribution: The present study shows that epistaxis is more common in first and second decades and once again the incidence increases after 4th decade; $30 \%$ cases were in the 1st and 2nd decades and 60\% cases from 4 th decade onwards. Amusa et $\mathrm{al}^{5}$. had a similar finding of around $40 \%$ cases uptil the 3 rd decade and $60 \%$ cases from 4 th decade onwards. Varshney et $\mathrm{al}^{6}$. also reported $73 \%$ cases in the 4 th decade and above.

Sex distribution: The present study shows $66 \%$ patients are males and $34 \%$ are females. The incidence in males is more as they are more exposed to trauma, assault and other injuries. Proportion of males and females in the present study is comparable to the above mentioned studies and exactly similar to that conducted by Hussain $^{7}$.

Seasonal distribution: In the present study, 58\% of cases are reported in the winter months and only $42 \%$ cases in summer and monsoon. This is in accordance with the findings of Monjas et al ${ }^{8}$. reported majority of cases in January to April. But in the study conducted by Phillip et $\mathrm{al}^{9}$. there was no winter predominance. The incidence is more during winter due to more frequent upper respiratory tract infection and due to dry weather. Due to these reasons, nasal mucosa becomes more inflamed and friable which leads to epistaxis.

Etiology: Recent study by Amusa et $\mathrm{al}^{5}$. showed traumatic epistaxis in $70.9 \%$ of cases. This can be explained on account of higher accident rate due to increase in the number of vehicles and bad roads and also increase in number of assault cases. The other major cause of epistaxis is hypertension which accounts for $30 \%$ of the cases. Most patients belong to the age group of 40 years and above. Hypertension was a major etiological factor in studies conducted by Juselius ${ }^{10}(47.3 \%)$, Monjas et $\mathrm{al}^{8}$. $(56 \%)$, Varshney et $\mathrm{al}^{6}$. (31.8\%). This due to the increase in lifestyle diseases.

Site: In the present study, $72 \%$ of cases have anterior epistaxis mainly from Little's area and lateral wall, probably of traumatic nature. $16 \%$ of cases have posterior epistaxis mainly due to hypertension. $12 \%$ of cases had both anterior and posterior bleeding. This is comparable to the study by Hussain et al ${ }^{7}$.

Treatment: In the present study $86 \%$ of the patients are treated by conservative measures like medical treatment $(42 \%)$, cautery $(6 \%)$, anterior nasal packing (30\%), posterior nasal packing (4\%) and foreign body removal (4\%). This is in accordance with Phillip et $\mathrm{al}^{9}$. study where $83 \%$ of the patients were treated successfully by non-interventional means. Similarly in the study by Urvashi et $\mathrm{al}^{11}$., almost $99 \%$ of cases were managed by conservative measures like cautery, anterior and posterior nasal packing.

$14 \%$ of the cases require surgical line of management which includes septal surgery $(6 \%)$, nasal bone fracture reduction (4\%), excision of bleeding polyp (2\%) and lateral rhinotomy with external carotid ligation 
(2\%). Similarly, surgical intervention was required in $17 \%$ of cases in Phillip et al ${ }^{9}$. study and $29.5 \%$ cases in Varshney et $\mathrm{al}^{6}$. study; mainly septal surgery and arterial ligation.

\section{Conclusion}

Epistaxis is a common clinical condition encountered by the otorhinolaryngologist. It is prevalent in the 1st and 2nd decade and once again the age incidence increases from the 4th decade onwards. It is found to be more common in males than females. It occurs frequently in cold and dry climate.

Anterior epistaxis is more common than posterior bleeds. The common causes epistaxis are trauma, hypertension, inflammatory conditions, septal abnormalities, bleeding disorders and idiopathic. Trauma and infection being more common in children and young adults, and hypertension and neoplasms in the elderly. intervention.

Majority of cases of epistaxis are manageable by conservative measures and only few require surgical

\section{References}

[1]. The American Heritage. Stedman's Medical Dictionary. Houghton Miffin Company; 2002.

[2]. Watkinson JC. Epistaxis. 6th ed. Chapter 18. In: Rhinology, Scott Brown's Otorhinolaryngology Head and Neck Surgery, Ian S Mackay, Bull TR, eds. Oxford: Butterworth Heinemann; 1998. pp. 942-61.

[3]. Gerald W McGarry. Epistaxis. 7th ed. Chapter 126. In: Scott Brown's Otorhinolaryngology Head and Neck Surgery, Michael Gleeson, ed. London: Hodder Arnold; 2008. pp. 1596-608.

[4]. Morgagni JB. The seats and causes of diseases. Investigated by Anatomy. Book 1; 1769. pp. 336-40.

[5]. Akinpelu OV, Amusa YB. A retrospective analysis of aetiology and management of epistaxis in a south-western Nigerian teaching hospital. West African Journal of Medicine 2009 May;28(3):165-8.

[6]. Sourabh Varshney, Saxena RK. Epistaxis: A retrospective clinical study. Indian Journal of Otrolaryngology and Head and Neck Surgery 2005 Apr-Jun;57:125-9.

[7]. Gulshan Hussain. Evaluation of aetiology and efficacy of management protocol of epistaxis. J Ayub Med Cell 2006;18(4).

[8]. Monjas-Canovas I. Epidemiology of epistaxis admitted to a tertiary hospital. Acta Otorrinolaringol Esp 2010 Jan-Feb;61(1):41-7.

[9]. Phillip A Pollice, Milton G Yude. A retrospective review of 249 hospitalised patients with epistaxis. AJO and Head and Neck Surgery 1997 Jul;49-53.

[10]. Juselius H. Epistaxis - A clinical study of 1724 patients. Journal of Laryngology and Otology; 1979. pp. $317-27$.

[11]. Urvashi Razdan, Raizada RM. Efficacy of conservative treatment modalities used in epistaxis. Indian Journal of Otolaryngology and Head and Neck Surgery 2004 Jan-Mar;56(1):20-3. 